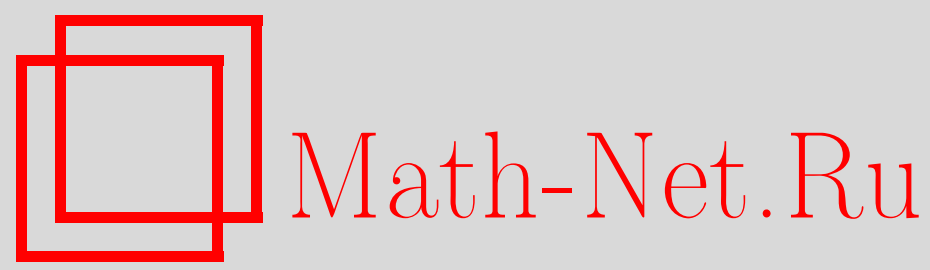

Ю. А. Дубинский, О задаче продолжения с наименьшим коаналитическим уклонением, Матем. заметки, 1998, том 64, выпуск 1, 45-57

DOI: https://doi.org/10.4213/mzm1371

Использование Общероссийского математического портала Math-Net.Ru подразумевает, что вы прочитали и согласны с пользовательским соглашением http://www.mathnet.ru/rus/agreement

Параметры загрузки:

IP: 18.234 .156 .22

26 апреля 2023 г., $12: 22: 54$

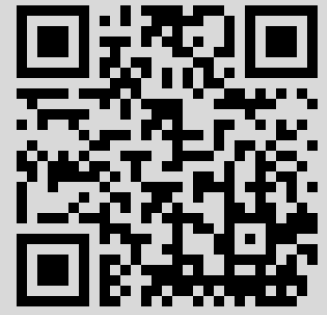




\section{О ЗАДАЧЕ ПРОДОЛЖЕНИЯ \\ С НАИМЕНЬШИМ КОАНАЛИТИЧЕСКИМ УКЛОНЕНИЕМ}

\section{Ю.А. Дубинский}

Рассматривается задача о нахождении такого продолжения функции с единичной окружности внутрь круга, чтобы оно наименее уклонялось от подпространства Соболева аналитических функций. Найдена математическая модель указанной задачи и установлена ее корректность.

Библиограбфия: 4 названия.

Введение. Пусть $K=\{z:|z|<1\}$ - единичньй круг на комплексной плоскости z, $S=\{z:|z|=1\}-$ его граница, и $f_{0}(s): s \rightarrow \mathbb{C}^{1}$ - некоторая функция.

В рамках теории соболевских пространств $W_{p}^{1}(K)$ рассматривается задача о нахождении такого продолжения граничной функции $f_{0}(s)$ внутрь круга $K$, которое наименее уклоняется от подпространства Соболева аналитических функций $\mathscr{O}_{p}^{1}(K) \subset W_{p}^{1}(K)$, $p>1$. Более точно, среди всевозможных функций $f(z): K \rightarrow \mathbb{C}^{1}$, таких, что $f(z) \in$ $W_{p}^{1}(K),\left.f(z)\right|_{S}=f_{0}(s)$, определяется та функция (наилучшее продолжение), для которой минимально "коаналитическое уклонение"

$$
\mu_{p}(f)=\left\|f(z)-f_{\mathrm{a}}(z)\right\|_{W_{p}^{1}(K)}^{p},
$$

где через $f_{\mathrm{a}}(z)$ обозначена аналитическая составляющая функции $f(z)$, соответствующая разложению $L_{p}(K)=\mathscr{O}_{p}(K) \oplus \partial_{z} \stackrel{\circ}{p}_{p}^{1}(K)$.

Математической моделью поставленной задачи является краевая задача для включения

$$
\begin{gathered}
-\Delta_{p}\left(f-f_{\mathrm{a}}\right)+\left|f-f_{\mathrm{a}}\right|^{p-2}\left(f-f_{\mathrm{a}}\right) \in \mathscr{O}_{p^{\prime}}^{-1}(K),\left.\quad f(z)\right|_{S}=f_{0}(s), \quad s \in S, \\
\left.P^{+}\left(\left|\nabla\left(f-f_{\mathrm{a}}\right)\right|^{p-2} \frac{\partial}{\partial n}\left(f-f_{\mathrm{a}}\right)\right)\right|_{S}=0,
\end{gathered}
$$

где $\Delta_{p}(u) \equiv \operatorname{div}\left(|\nabla u|^{p-2} \nabla u\right)$ есть $p$-лапласиан, $\mathscr{O}_{p^{\prime}}^{-1}(K)=\left(\mathscr{O}_{p}^{1}(K)\right)^{*}-$ пространство аналитических функционалов, $P^{+}-$проектор на подпространство функций на единичной окружности $S$, ряд Фурье которых состоит только из экспонент с положительными частотами, т.е. на подпространство функций вида

$$
f(\Theta)=\sum_{n \geqslant 0} f_{n} \exp (i n \Theta)
$$

Работа выполнена при финансовой поддержке Российского фонда фундаментальных исследований, грант № 96-01-00621. 
$(\Theta$ - полярньй угол); наконец, $\partial / \partial n$ - дифференцирование по нормали к $S$.

Устанавливается корректность поставленной задачи и, тем самым, корректность исходной задачи о существовании такого продолжения функции $f_{0}(s)$, которое имеет в $W_{p}^{1}$-норме наименьшую коаналитическую часть $f_{\text {са }}(z) \equiv f(z)-f_{\mathrm{a}}(z)$.

В случае $p=2$ и “чисто" градиентной метрики, т.е. для $\mu_{2}(f)=\left\|\nabla\left(f-f_{\mathrm{a}}\right)\right\|_{L_{2}(K)}^{2}$, наилучшим продолжением, как оказывается, является гармоническое продолжение.

Интересно отметить, что в случае кольца картина иная. В этом случае гармоническое продолжение граничных функций не совпадает с продолжением, имеющим наименьшее коаналитическое уклонение.

\section{1. Разложение пространства Лебега в прямую сумму аналитического и} коаналитического подпространств.

1.1. Пространство $\mathscr{O}_{p}(K)$. Базисность системы $z^{n}, n=0,1, \ldots$ Результаты данного подпункта носят вспомогательньй характер.

Пусть

$$
L_{p}(K)=\left\{f(z): K \rightarrow \mathbb{C}^{1},\|f\|_{p}^{p}=\iint_{K}|f|^{p} d x d y<\infty\right\}
$$

- пространство Лебега в круге $K$. Далее, обозначим через $\mathscr{O}_{p}(K)$ подпространство Бергмана аналитических в круге $K$ функций из $L_{p}$, т.е.

$$
\mathscr{O}_{p}(K)=\left\{f(z) \in L_{p}(K): \partial_{\bar{z}} f(z) \equiv 0\right\}
$$

где, как обычно, $\partial_{\bar{z}}-$ оператор Коши-Римана.

ПРЕДЛОЖЕНИЕ 1. Система мономов $z^{n}, n=0,1, \ldots$, является базисом Фурье в $\mathscr{O}_{p}(K)$, т.е. любая функиия $f(z) \in \mathscr{O}_{p}(K)$ представима в виде сходящегося в $L_{p}$-норме ряда

$$
f(z)=\sum_{n=0}^{\infty} c_{n} z^{n}
$$

$2 \partial e$

$$
c_{n}=\frac{\left\langle f(z), \bar{z}^{n}\right\rangle_{K}}{\left\|z^{n}\right\|_{2}^{2}}=\frac{n+1}{\pi} \iint_{K} f(z) \bar{z}^{n} d x d y .
$$

ДокАЗАТЕЛЬСтво. Действительно, в силу аналитичности

$$
f(z)=\sum_{n=0}^{\infty} c_{n} z^{n} \equiv \sum_{n=0}^{\infty} c_{n} \rho^{n} e^{i n \Theta}
$$

при любом фиксированном $\rho \in(0,1)$ имеем

$$
c_{n} \rho^{n}=\frac{1}{2 \pi} \int_{0}^{2 \pi} f\left(\rho e^{i \Theta}\right) e^{-i n \Theta} d \Theta
$$

или, что то же,

$$
c_{n} \rho^{2 n}=\frac{1}{2 \pi} \int_{0}^{2 \pi} f(z) \bar{z}^{n} d \Theta
$$


Интегрируя последнее равенство по мере $\rho d \rho$, немедленно получаем, что $c_{n}$ определяется формулой (2) и, тем самым, ряд (1) является рядом Фурье функции $f(z)$ в пространстве $L_{p}(K)$.

Заметим далее, что при каждом $\rho \in(0,1)$ ряд $(3)$ сходится к $f(z)$ равномерно и, следовательно, в силу известной $L_{p}$-оценки частичных сумм ряда $Ф$ урье (см., например, $[1$, с. 594$])$

$$
\left\|\sum_{n=0}^{N} c_{n} z^{n}\right\|_{L_{p}(0,2 \pi)} \leqslant M_{p}\|f(z)\|_{L_{p}(0,2 \pi)}, \quad N=1,2, \ldots,
$$

где $M_{p}>0$ - постоянная, зависящая только от $p>1$. Тем самьм, в соответствии с классической теоремой Лебега о предельном переходе под знаком интеграла ряд (1) сходится к функции $f(z)$ в норме $L_{p}(K)$.

Предложение 1 доказано.

1.2. Формула $L_{p}(K)=\mathscr{O}_{p}(K) \oplus \partial_{z} \stackrel{\circ}{W}_{p}^{1}(K)$. С полученным разложением $(1),(2)$ естественно связать оператор, действующий из пространства $L_{p}(K)$ в пространство $\mathscr{O}_{p}(K)$. Именно, каждой функции $f(z) \in L_{p}(K)$ сопоставим аналитическую функцию, определяемую рядом

$$
f_{\mathrm{a}}(z)=\sum_{n=0}^{\infty} c_{n} z^{n}
$$

где коэффищиенты $c_{n}$ вычислены по формулам (2).

Подставляя в этот ряд формулы $(2)$, легко видеть, что

$$
f_{\mathrm{a}}(z)=\frac{1}{\pi} \iint_{K} \frac{f(w) d u d v}{(1-z \bar{w})^{2}}, \quad w=u+i v,
$$

т.е. отображение $f(z) \mapsto f_{\mathrm{a}}(z)$ есть классический ограниченный проектор Бергмана на подпространство $\mathscr{O}_{p}(K)$ (см., например, обзоры [2] и [3]). Для замкнутости изложения мы приводим доказательство этого факта в п. 5.

Будем назьвать функцию $f_{\mathrm{a}}(z) \in \mathscr{O}_{p}(K)$ аналитической частью исходной функции $f(z) \in L_{p}(K)$. Функцию $f_{\text {са }}(z)=f(z)-f_{\mathrm{a}}(z)$ назовем коаналитической частью функции $f(z)$.

Таким образом, пространство $L_{p}(K)$ разлагается в прямую сумму

$$
L_{p}(K)=\mathscr{O}_{p}(K) \oplus \mathscr{O}_{p}^{\perp}(K)
$$

где по определению

$$
\mathscr{O}_{p}^{\perp}(K)=\left\{q(z) \in L_{p}(K): q(z)=f(z)-f_{\mathrm{a}}(z), f(z) \in L_{p}(K)\right\}
$$

- подпространство коаналитических функций.

Из предложения 1 ясно, что $q(z) \in \mathscr{O}_{p}^{\perp}(K)$ тогда и только тогда, когда

$$
\left\langle q(z), z^{n}\right\rangle_{K}=0, \quad n=0,1, \ldots
$$

Наша ближайшая цель - описать подпространство $\mathscr{O}_{p}^{\perp}(K)$. Имеет место следующая 
Теорема 1. Справедлива формула

$$
\mathscr{O}_{p}^{\perp}(K)=\partial_{z} \stackrel{\circ}{p}_{p}^{1}(K), \quad \partial_{z}=\frac{1}{2}\left(\frac{\partial}{\partial x}-i \frac{\partial}{\partial y}\right),
$$

где $\stackrel{\circ}{W}_{p}^{1}(K)$ - пространство Соболева функций, обращающихся в нуль на граниче круга. Более точно, функиия $q(z) \in \mathscr{O}_{p}^{\perp}(K)$ тогда и только тогда, когда существует (единственная) функция $r(z) \in \stackrel{\circ}{W}_{p}^{1}(K)$ такая, что $q(z)=\partial_{z} r(z)$. При этом выполнена оценка

$$
\|r(z)\|_{p}+\|\nabla r(z)\|_{p} \leqslant M\|q(z)\|_{p},
$$

әде $M>0$ - постоянная.

ДокАЗАТЕЛЬСтво. Достаточность очевидна. Действительно, если $q(z)=\partial_{z} r(z)$, где $r(z) \in \stackrel{\circ}{W}_{p}^{1}(K)$, то, интегрируя по частям, имеем

$$
\left\langle q(z), z^{n}\right\rangle_{K}=\left\langle\partial_{z} r(z), z^{n}\right\rangle_{K}=-\left\langle r(z), \partial_{\bar{z}} z^{n}\right\rangle_{K}=0, \quad n=0,1, \ldots
$$

Это значит, что $q(z) \in \mathscr{O}_{p}^{\perp}(K)$.

Обратно, пусть функция $r(z)$ является решением краевой задачи

$$
\begin{aligned}
\frac{1}{2}\left(\frac{\partial}{\partial x}-i \frac{\partial}{\partial y}\right) r(z) & =q(z), \quad z \in K, \\
\left.r(z)\right|_{S} & =0,
\end{aligned}
$$

которое в полярных координатах $\rho, \Theta$ формально имеет вид

$$
r(z)=\sum_{n=-\infty}^{+\infty} r_{n}(\rho) e^{i n \Theta}
$$

где

$$
r_{n}(\rho)=\left\{\begin{array}{c}
2 \int_{0}^{\rho} \eta^{n} q_{n-1}(\eta) d \eta \rho^{-n}, \quad n>0, \\
-2 \int_{\rho}^{1} \eta^{n} q_{n-1}(\eta) d \eta \rho^{-n}, \quad n \leqslant 0,
\end{array}\right.
$$

а $q_{n}(\rho)$ - соответствуюшая проекция $q(z)$ в разложении в ряд Фурье по угловой переменной $\Theta$.

Легко видеть, что условия "ортогональности" (4) эквивалентны равенствам

$$
\int_{0}^{1} \rho^{n} q_{n-1}(\rho) d \rho=0, \quad n=1,2, \ldots,
$$

откуда немедленно следует, что $r_{n}(1)=0$ для всех $n=0, \pm 1, \ldots$ Тем самым, ряд (6) является формальным решением задачи Дирихле (5)

$$
\Delta r(z)=\partial_{\bar{z}} q(z),\left.\quad r\right|_{S}=0,
$$

и, следовательно, в соответствии с известной теорией $L_{p}$-оценок решений коэрцитивных эллиптических задач имеет место оценка

$$
\|\nabla r(z)\|_{p}+\|r(z)\|_{p} \leqslant M\|q(z)\|_{p},
$$

где $M>0$ - постоянная.

Это означает, что частичные суммы ряда (6), являясь, очевидно, неформальньми решениями задачи (5) (при соответствуюших правых частях), сходятся в $W_{p}^{1}$-норме и, тем самым, определяют искомое решение.

Теорема 1 доказана. 
ЗАмЕчАНИЕ. Нетрудно подсчитать, что в интегральной форме решение имеет вид

$$
r(z)=\frac{1}{\pi} \iint_{K} \frac{f(\zeta) d \xi d \eta}{\bar{z}-\bar{\zeta}}, \quad \zeta=\xi+i \eta
$$

2. Коаналитическое уклонение. Постановка основной задачи. Как вытекает из п. 1 , всякая функция $f(z) \in W_{p}^{1}(K)$ представляется как функция из $L_{p}(K)$ в виде суммы аналитической и коаналитической составляющих: $f(z)=f_{\mathrm{a}}(z)+f_{\text {са }}(z)$. Поэтому естественно считать ее коаналитическую составляющую $f_{\text {са }}(z)$ определенной характеристикой неаналитичности самой функции $f(z)$.

ОПРЕДЕЛЕНИЕ. Мерой неаналитичности или, что то же, коаналитическим уклонением функиии $f(z) \in W_{p}^{1}(K)$ назовем число

$$
\begin{aligned}
\mu_{p}(f) & =\left\|f_{\mathrm{ca}}(z)\right\|_{W_{p}^{1}(K)}^{p} \equiv\left\|\nabla\left(f-f_{\mathrm{a}}\right)\right\|_{L_{p}}^{p}+\left\|f-f_{\mathrm{a}}\right\|_{L_{p}}^{p} \\
& \equiv \iint_{K}\left(\left(\left|\frac{\partial}{\partial x}\left(f-f_{\mathrm{a}}\right)\right|^{2}+\left|\frac{\partial}{\partial y}\left(f-f_{\mathrm{a}}\right)\right|^{2}\right)^{p / 2}+\left|f-f_{\mathrm{a}}\right|^{p}\right) d x d y .
\end{aligned}
$$

ЗАмЕчАниЕ. Естественно, давая это определение, мы должны показать, что если $f(z) \in W_{p}^{1}(K)$, то ее аналитическая часть $f_{\mathrm{a}}(z)$ (а, значит, и коаналитическая часть) также принадлежат пространству $W_{p}^{1}(K)$. Действительно, если

то

$$
f_{\mathrm{a}}(z)=\sum_{n=0}^{\infty} f_{n} z^{n}
$$

где

$$
f_{\mathrm{a}}^{\prime}(z)=\sum_{n=1}^{\infty} n f_{n} z^{n-1}=\sum_{n=1}^{\infty} c_{n-1} z^{n-1},
$$

$$
c_{n-1}=\frac{n(n+1)}{\pi} \iint_{K} f(z) \bar{z}^{n} d x d y .
$$

Переходя к полярным координатам $\rho, \Theta$ и интегрируя по частям по угловой переменной, получим, что

где

$$
c_{n-1}=\frac{n+1}{\pi} \iint_{K} g(z) \bar{z}^{n-1} d x d y,
$$

$$
g(z) \equiv-i\left(-\frac{\partial f}{\partial x} \sin \Theta+\frac{\partial f}{\partial y} \cos \Theta\right) \bar{z}^{2} e^{i \Theta} \in L_{p}(K) .
$$

Тем самым, $c_{n-1}=(n+1) g_{n-1} / n$, где $g_{n-1}-$ коэффициенты Фурье функции $g_{\mathrm{a}}(z)$ в разложении по системе $z^{n}, n=0,1, \ldots$, т.е.

$$
g_{\mathrm{a}}(z)=\sum_{n=0}^{\infty} g_{n} z^{n}
$$

(ряд сходится в норме $L_{p}(K)$ ).

Рассматривая этот ряд как ряд Фурье функции $g_{\mathrm{a}}\left(\rho e^{i \Theta}\right), \rho \in(0,1)$, по системе экспонент и применяя классический результат Марцинкевича к последовательности $\lambda_{n}=$ $(n+1) / n$ (см., например, $[4$, с. 345$])$, получаем, что ряд (7) также сходится в $L_{p}(K)$, что и требуется.

Таким образом, определение меры неаналитичности корректно.

С учетом этого определения обратимся к постановке задачи продолжения функции $f_{0}(s) \in W_{p}^{1 / p^{\prime}}(S)$ внутрь единичного круга. 
ЗАДАчА. Среди всевозможных продолжений $f(z) \in W_{p}^{1}(K),\left.f(z)\right|_{S}=f_{0}(s)$, найти то, которое имеет наименьшее коаналитическое уклонение $\mu_{p}(f)$.

Иными словами, ставится задача минимизации $\left\|f_{\text {са }}(z)\right\|_{W_{p}^{1}(K)}^{p} \rightarrow \min$, где $f(z) \in$ $W_{p}^{1}(K)$ - всевозможные продолжения фиксированной функции $f_{0}(s) \in W_{p}^{1 / p^{\prime}}(S)$.

Получим математическую модель поставленной задачи. Действительно, как известно, в точке минимума вариация функционала $\mu_{p}(f)$ равна 0 , т.е. для любой функции $\varphi(z) \in \stackrel{\circ}{W}_{p}^{1}(K)$ имеем

$$
\begin{aligned}
L(f) \varphi \equiv & \iint_{K}\left(\left|\nabla\left(f-f_{\mathrm{a}}\right)\right|^{p-2} \nabla\left(f-f_{\mathrm{a}}\right) \nabla\left(\bar{\varphi}-\bar{\varphi}_{\mathrm{a}}\right)\right. \\
& \left.+\left|f-f_{\mathrm{a}}\right|^{p-2}\left(f-f_{\mathrm{a}}\right)\left(\bar{\varphi}-\bar{\varphi}_{\mathrm{a}}\right)\right) d x d y=0,
\end{aligned}
$$

где $\varphi(z) \in \stackrel{\circ}{W}_{p}^{1}(K)-$ произвольная функция.

Выберем в (8) в качестве $\varphi(z)$ любую функцию вида $\varphi(z)=\partial_{z} r(z)$, где $r(z) \in W_{p}^{2}(K)$. Тогда, интегрируя по частям, немедленно получим

$$
\begin{aligned}
& \left\langle-\Delta_{p}\left(f-f_{\mathrm{a}}\right)+\left|f-f_{\mathrm{a}}\right|^{p-2}\left(f-f_{\mathrm{a}}\right), \varphi(z)-\varphi_{\mathrm{a}}(z)\right\rangle_{K} \\
& \quad \equiv \iint_{K}\left(-\Delta_{p}\left(f-f_{\mathrm{a}}\right)+\left|f-f_{\mathrm{a}}\right|^{p-2}\left(f-f_{\mathrm{a}}\right)\right) \overline{\varphi(z)} d x d y=0
\end{aligned}
$$

ибо в выбранном случае $\varphi_{\mathrm{a}}(z) \equiv 0$.

Отсюда, предполагая (временно), что выражение в квадратных скобках суммируемо со степенью $p^{\prime}=p /(p-1)$ и учитьвая плотность выбранных функций $\varphi(z)$ в $\mathscr{O}_{p}^{\perp}(K)$, приходим к включению

$$
-\Delta_{p}\left(f-f_{\mathrm{a}}\right)+\left|f-f_{\mathrm{a}}\right|^{p-2}\left(f-f_{\mathrm{a}}\right) \in \mathscr{O}_{p^{\prime}}(K) .
$$

Для вьвода граничного условия положим теперь в тождестве (8) произвольно $\varphi(z) \in$ $W_{p}^{1}(K)$. Тогда, интегрируя по частям, придем к равенству

$$
\begin{array}{r}
\left\langle-\Delta_{p}\left(f-f_{\mathrm{a}}\right)+\left|f-f_{\mathrm{a}}\right|^{p-2}\left(f-f_{\mathrm{a}}\right), \varphi(z)-\varphi_{\mathrm{a}}(z)\right\rangle_{K} \\
+\int_{S}\left|\nabla\left(f-f_{\mathrm{a}}\right)\right|^{p-2} \frac{\partial}{\partial n}\left(f-f_{\mathrm{a}}\right)\left(\bar{\varphi}-\bar{\varphi}_{\mathrm{a}}\right) d S=0
\end{array}
$$

или, учитьвая только что вьведенное включение (9) и условие $\left.\varphi\right|_{S}=0$, к равенству

$$
\int_{S}\left|\nabla\left(f-f_{\mathrm{a}}\right)\right|^{p-2} \frac{\partial}{\partial n}\left(f-f_{\mathrm{a}}\right) \bar{\varphi}_{\mathrm{a}} d S=0 .
$$

В частности, фиксируя индекс $n=0,1, \ldots$, выберем $\varphi(z) \equiv \varphi_{n}(z)=R(\rho) \exp (i n \Theta)$, где $R(\rho) \in C_{0}^{\infty}(0,1)$ такова, что

$$
\int_{0}^{1} R(\rho) \rho^{n+1} d \rho \neq 0
$$


Тогда (10) немедленно приводит к набору условий

$$
\int_{-\pi}^{\pi}\left(\left|\nabla\left(f-f_{\mathrm{a}}\right)\right|^{p-2} \frac{\partial}{\partial n}\left(f-f_{\mathrm{a}}\right)\right)_{\rho=1} e^{-i n \Theta} d \Theta=0, \quad n=0,1, \ldots
$$

Это означает, что в разложении (вообще говоря, в смысле обобщенных функций) периодической функции

$$
\left(\left|\nabla\left(f-f_{\mathrm{a}}\right)\right|^{p-2} \frac{\partial}{\partial n}\left(f-f_{\mathrm{a}}\right)\right)_{\rho=1}
$$

в ряд Фурье по угловой переменной все слагаемые, отвечающие положительным частотам, равны 0.

Если обозначить через $P^{+}: L_{p^{\prime}}(-\pi, \pi) \rightarrow L_{p^{\prime}}^{+}(-\pi, \pi)$ проектор на подпространство периодических функций, разлагающихся в ряд по $\exp ($ in $\Theta), n=0,1, \ldots$, то полученное условие принимает вид

$$
P^{+}\left(\left.\left|\nabla\left(f-f_{\mathrm{a}}\right)\right|^{p-2} \frac{\partial}{\partial n}\left(f-f_{\mathrm{a}}\right)\right|_{\varrho=1}\right)=0
$$

что и требовалось.

Таким образом, в итоге находим, что решение $f(z)$ поставленной задачи удовлетворяет дифференциальному включению (9) и граничным условиям

$$
\left.f(z)\right|_{S}=f_{0}(s), \quad s \in S
$$

и (11), последнее из которых носит проекционньй характер.

Напомним, однако, что эта модель получена нами при дополнительном условии $-\Delta_{p}\left(f-f_{\mathrm{a}}\right)+\left|f-f_{\mathrm{a}}\right|^{p-2}\left(f-f_{\mathrm{a}}\right) \in L_{p^{\prime}}(K)$. В общем случае этого включения может не быть и, строго говоря,

$$
L(f) \equiv-\Delta_{p}\left(f-f_{\mathrm{a}}\right)+\left|f-f_{\mathrm{a}}\right|^{p-2}\left(f-f_{\mathrm{a}}\right) \in W_{p^{\prime}}^{-1}(K),
$$

т.е. является функционалом над пространством $W_{p}^{1}(K)$. Этот функционал можно, очевидно, представить в виде $L(f)=L_{\mathrm{a}}(f)+L_{\text {са }}(f)$, где аналитический функционал $L_{\mathrm{a}}(f)$ действует по формуле

$$
L_{\mathrm{a}}(f) \varphi_{\mathrm{a}}(z)=\iint_{K}\left(\left|\nabla\left(f-f_{\mathrm{a}}\right)\right|^{p-2} \nabla\left(f-f_{\mathrm{a}}\right) \nabla \overline{\varphi_{\mathrm{a}}}+\left|f-f_{\mathrm{a}}\right|^{p-2}\left(f-f_{\mathrm{a}}\right) \overline{\varphi_{\mathrm{a}}}\right) d x d y
$$

а коаналитический функционал $L_{\text {са }}(f)$ - по аналогичной формуле, в которой $\varphi_{\mathrm{a}}(z)$ заменяется на $\varphi(z)-\varphi_{\mathrm{a}}(z)$. Поскольку $L_{\text {са }}(f)=0$, то $L(f)=L_{\mathrm{a}}(f)$, т.е. оператор $-\Delta_{p}\left(f-f_{\mathrm{a}}\right)+\left|f-f_{\mathrm{a}}\right|^{p-2}\left(f-f_{\mathrm{a}}\right)$ определяет на решении $f(z)$ функционал, которьй можно отождествить с функционалом над подпространством аналитических функций $\mathscr{O}_{p}^{1}(G)$. Будем обозначать сказанное включением

$$
-\Delta_{p}\left(f-f_{\mathrm{a}}\right)+\left|f-f_{\mathrm{a}}\right|^{p-2}\left(f-f_{\mathrm{a}}\right) \in \mathscr{O}_{p^{\prime}}^{-1}(K) .
$$

В конечном итоге математическая модель задачи о наилучшем продолжении граничной функции $f_{0}(s) \in W_{p}^{1 / p^{\prime}}(S)$ составляет задачу о решении включения (13) при условиях $(12),(11)$. Последнее условие понимается в смысле теории следов обобщенных функций со значениями в пространстве $W_{p^{\prime}}^{-1}(-\pi, \pi)$. 
3. Существование и единственность наилучшего продолжения. Имеет место основная

ТЕорема 2. Для любой функиии $f_{0}(s) \in W_{p}^{1 / p^{\prime}}(S)$ существует единственное решение $f(z) \in W_{p}^{1}(K)$ задачи (11)-(13) или, что то жсе, задачи минимизачии коаналитического уклонения.

ДокАзАТЕЛьСтво. Доказательство как существования, так и единственности решения проводится в рамках идей теории монотонных операторов.

Сушествование. Сведем, прежде всего, задачу к случаю нулевой функции на границе. Именно, пусть $h(z) \in W_{p}^{1}(K)$ - какое-либо фиксированное продолжение функции $f_{0}(s)$, т.е. $\left.h(z)\right|_{S}=f_{0}(s)$. Положим $u(z)=f(z)-h(z)$. Тогда функция $u(z) \in W_{p}^{1}(K)$ удовлетворяет интегральному тождеству (см. (8)) $L(u+h) \varphi(z)=0$, где $\varphi(z) \in W_{p}^{1}(K)$, $\left.\varphi(z)\right|_{S}=0$, произвольна.

Пусть $\varphi^{1}(z), \varphi^{2}(z), \ldots$ - базисная система в пространстве $\stackrel{\circ}{W}_{p}^{1}(K)$. Приближенное решение $u^{N}(z), N=1,2, \ldots$, ищем в виде

$$
u^{N}(z)=C_{N}^{1} \varphi^{1}(z)+\cdots+C_{N}^{N} \varphi^{N}(z)
$$

где неизвестные постоянные $C_{N}^{1}, \ldots, C_{N}^{N}$ определяются из нелинейной системы моментных уравнений Галёркина

$$
L\left(u^{N}+h\right) \varphi^{j}(z)=0, \quad j=1, \ldots, N
$$

В силу свойства монотонности оператора $L$ система (14) однозначно разрешима. При этом для коаналитических составляющих $u_{\mathrm{ca}}^{N}(z)$ имеем оценку

$$
\left\|u_{\mathrm{ca}}^{N}(z)\right\|_{W_{P}^{1}(K)} \leqslant M
$$

где $M>0$ - постоянная, не зависящая от $N$.

Нетрудно заметить, что и для аналитических составляющих $u_{\mathrm{a}}^{N}(z)$ справедлива аналогичная оценка. Действительно, из оценки (15) и точных неравенств между нормами функций в круге $K$ и их следов на окружности $S$ следует, что для всех $N$

$$
\left\|\left.u_{\mathrm{ca}}^{N}(z)\right|_{S}\right\|_{W_{p}^{1 / p^{\prime}}(S)} \leqslant M\left\|u_{\mathrm{ca}}^{N}(z)\right\|_{W_{p}^{1}(K)} \leqslant M_{1}
$$

$M_{1}>0$ - постоянная. Отсюда, учитьвая, что $\left.u_{\mathrm{a}}^{N}(z)\right|_{S}+\left.u_{\mathrm{ca}}^{N}(z)\right|_{S}=0$, находим, что и

$$
\left\|\left.u_{\mathrm{a}}^{N}(z)\right|_{S}\right\|_{W_{p}^{1 / p^{\prime}}(S)} \leqslant M_{2}
$$

где $M_{2}>0$ не зависит от $N$.

Остается заметить, что в силу известных $L_{p}$-оценок теории эллиптических задач

$$
\left\|u_{\mathrm{a}}^{N}(z)\right\|_{W_{p}^{1}(K)} \leqslant M_{3}\left\|\left.u_{\mathrm{a}}^{N}(z)\right|_{S}\right\|_{W_{p}^{1 / p^{\prime}}(S)} \leqslant M_{4},
$$

где $M_{4}>0$ - постоянная, ибо всякая аналитическая функция является и гармонической (комплекснозначной) функцией. 
Таким образом, в конечном итоге получаем оценку

$$
\left\|u^{N}(z)\right\|_{W_{p}^{1}(K)} \leqslant M_{5}
$$

где $M_{5}>0$ не зависит от $N$.

Далее обычными рассуждениями устанавливаем, что предельная точка $u(z)$ последовательности $u^{N}(z)$ удовлетворяет интегральному тождеству (14). Существование решения доказано.

Единственность. Действительно, пусть $f(z) \in W_{p}^{1}(K)$ и $g(z) \in W_{p}^{1}(K)$ - два решения задачи (11)-(13). Тогда, исходя из тождества (8), стандартно получим, что их коаналитические составляющие равны, т.е. $f_{\text {са }}(z) \equiv g_{\text {са }}(z)$. Отсюда, учитьвая, что $\left.(f(z)-g(z))\right|_{S}=0$, получаем, что $\left.f_{\mathrm{a}}(z)\right|_{S}=\left.g_{\mathrm{a}}(z)\right|_{S}$. Следовательно, $f_{\mathrm{a}}(z) \equiv g_{\mathrm{a}}(z)$, $z \in K$, и тем самым, $f(z) \equiv g(z), z \in K$. Единственность, а вместе с ней и теорема 2 полностью доказаны.

4. Случай градиентной метрики. В качестве конкретного примера рассмотрим случай среднеквадратичного коаналитического уклонения в “чисто" градиентной метрике

$$
\mu_{2}(f)=\iint_{K}\left|\nabla\left(f(z)-f_{\mathrm{a}}(z)\right)\right|^{2} d x d y .
$$

Математическая модель этой задачи имеет вид

$$
\begin{gathered}
\Delta f(z) \in \mathscr{O}_{2}^{-1}(K), \\
\left.f(z)\right|_{S}=f_{0}(s), \quad P^{+}\left(\left.\frac{\partial}{\partial n}\left(f-f_{\mathrm{a}}\right)\right|_{S}\right)=0 .
\end{gathered}
$$

Эта задача имеет единственное решение $f(z) \in W_{2}^{1}(K)$ для любой функции $f_{0}(s) \in$ $W_{2}^{1 / 2}(S)$. Более того, решение может быть найдено стандартно методом Фурье в полярных координатах. Элементарньй, но довольно длинньй подсчет показьвает, что решением оказывается обычное гармоническое продолжение, т.е. решение задачи Дирихле

$$
\Delta f(z)=0,\left.\quad f(z)\right|_{S}=f_{0}(s), \quad s \in S .
$$

Мы не станем проводить этот подсчет, а докажем непосредственно эквивалентность этих задач.

ПРЕДЛОЖЕНИЕ 2. Тождество

$$
\left\langle\nabla\left(f-f_{\mathrm{a}}\right), \nabla\left(\varphi-\varphi_{\mathrm{a}}\right)\right\rangle_{K}=\langle\nabla f, \nabla \varphi\rangle_{K} \quad \forall \varphi(z) \in \stackrel{\circ}{W}_{2}^{1}(K)
$$

имеет место тогда и только тогда, когда

$$
P^{+}\left(\left.\left(f-f_{\mathrm{a}}\right)\right|_{S}\right)=0
$$


ДокАЗАТЕЛЬСТво. Ясно, что (18) достаточно установить для функций $\varphi(z)$, гладких и финитных в круге $K$. Имеем, интегрируя по частям,

$$
\begin{aligned}
\left\langle\nabla\left(f-f_{\mathrm{a}}\right), \nabla\left(\varphi-\varphi_{\mathrm{a}}\right)\right\rangle_{K} & =\langle\nabla f, \nabla \varphi\rangle_{K}-\left\langle\nabla\left(f-f_{\mathrm{a}}\right), \nabla \varphi_{\mathrm{a}}\right\rangle_{K}-\left\langle\nabla f_{\mathrm{a}}, \nabla \varphi\right\rangle_{K} \\
& =\langle\nabla f, \nabla \varphi\rangle_{K}-\int_{S}\left(f-f_{\mathrm{a}}\right) \frac{\partial}{\partial n} \varphi_{\mathrm{a}} d s,
\end{aligned}
$$

ибо $\Delta f_{\mathrm{a}}=0$ и $\Delta \overline{\varphi_{\mathrm{a}}}=0$ в $K$. Это значит, что тождество (18) справедливо тогда и только тогда, когда

$$
\int_{S}\left(f-f_{\mathrm{a}}\right) \frac{\partial}{\partial n} \varphi_{\mathrm{a}} d s=0 .
$$

Выбрав теперь последовательность функций $\varphi(z) \equiv \varphi\left(\varrho e^{i \Theta}\right)=q(\varrho) \exp (i n \Theta), n=0$, $1, \ldots$, где $q(\varrho) \in C_{0}^{\infty}(0,1)$ и такова, что

$$
\int_{0}^{1} q(\varrho) \varrho^{n+1} d \varrho \neq 0
$$

немедленно получаем, что

$$
\int_{0}^{2 \pi}\left(f-f_{\mathrm{a}}\right) \exp (i n \Theta) d \Theta=0, \quad n=0,1, \ldots
$$

Это значит, что выполнено (19). Обратное очевидно.

Предложение 2 доказано.

ПРЕДЛОЖЕНИЕ 3. Пусть $f(z) \in W_{2}^{1}(K)$ - решение задачи минимизации

$$
\mu_{2}(f) \rightarrow \inf
$$

Тогда выполнено (19).

ДокАЗАТЕЛьСтво. Действительно,

$$
\left\langle\nabla\left(f-f_{\mathrm{a}}\right), \nabla\left(\varphi-\varphi_{\mathrm{a}}\right)\right\rangle_{K}=\left\langle f-f_{\mathrm{a}}, \Delta \varphi\right\rangle_{K}+\int_{S}\left(f-f_{\mathrm{a}}\right) \frac{\partial}{\partial n}\left(\varphi-\varphi_{\mathrm{a}}\right) d s=0 .
$$

Выберем в качестве функции $\varphi(z)$ решение задачи

$$
\Delta \varphi(z)=z^{n},\left.\quad \varphi(z)\right|_{S}=0, \quad n=0,1, \ldots
$$

Это будет последовательность

$$
\varphi(z)=\frac{1}{4(n+1)}\left(|z|^{2}-1\right) z^{n}
$$

для которой

$$
\left.\frac{\partial}{\partial n}\left(\varphi(z)-\varphi_{\mathrm{a}}(z)\right)\right|_{S}=\mathscr{A}_{n} e^{i n \Theta}, \quad \mathscr{A}_{n} \neq 0 .
$$

Следовательно, учитьвая, что $f(z)-f_{\mathrm{a}}(z) \in \mathscr{O}_{2}^{\perp}(G)$, из (20) немедленно получаем (19). Предложение 3 доказано.

Следствием предложений 2, 3 и является эквивалентность задач (16) и (17). Тем самым, гармоническое продолжение минимизирует не только функционал Дирихле, но и функционал коаналитической невязки $\mu_{2}(f)$.

Как уже отмечалось, аналогичное утверждение для кольца не имеет места. 
5. Дополнение. В этом пункте устанавливается ограниченность аналитического проектора $f(z) \mapsto f_{\mathrm{a}}(z)$ в пространствах $L_{p}(K), p>1$ - произвольное число.

Напомним, что

$$
f_{\mathrm{a}}(z)=\sum_{n=0}^{\infty} c_{n} z^{n}, \quad \text { где } \quad c_{n}=\frac{n+1}{\pi} \iint_{K} f(z) \bar{z}^{n} d x d y
$$

ПРЕДЛОЖЕНИЕ 4. Имеет место неравенство

$$
\left\|f_{\mathrm{a}}(z)\right\|_{L_{p}(K)} \leqslant M\|f(z)\|_{L_{p}(K)}
$$

әде $M>0$ - постоянная, зависящая только от $p>1$.

ДокАЗАТЕЛЬСТВо. Пусть $r \in(0,1)$ - некоторое число, и функция $f(z)$ представлена в виде $f(z)=f_{0}^{r}(z)+f_{r}^{1}(z)$, где $f_{0}^{r}(z)=f(z) \chi_{(0, r)}(|z|), f_{r}^{1}(z)=f(z) \chi_{(r, 1)}(|z|)\left(\chi_{E}(t)\right.$, как обычно, - характеристическая функция множества $E$ ). Тогда элементарньй подсчет, исходя из определения $f_{\mathrm{a}}(z)$, показывает, что

$$
\left\|f_{\mathrm{a}}(z)\right\|_{L_{p}\left(K_{0}^{r}\right)} \leqslant M\|f(z)\|_{L_{p}(K)}, \quad M>0
$$

где $K_{0}^{r}=\{z:|z|<r\}$. Точно так же

$$
\left\|\left(f_{0}^{r}(z)\right)_{\mathrm{a}}\right\|_{L_{p}(K)} \leqslant M\left\|f_{0}^{r}(z)\right\|_{L_{p}\left(K_{0}^{r}\right)} \equiv M\left\|f_{0}^{r}(z)\right\|_{L_{p}(K)} .
$$

Таким образом, дело сводится к доказательству неравенства

$$
\left\|f_{\mathrm{a}}(z)\right\|_{L_{p}\left(K_{r}^{1}\right)} \leqslant M\|f(z)\|_{L_{p}\left(K_{r}^{1}\right)}
$$

для функций $f(z) \in L_{p}(K)$, носитель которых содержится в кольце $K_{r}^{1}=\{z: r<|z|<1\}$.

С этой целью положим $\varrho_{j}=e^{-j h}$, где $j=0,1, \ldots, N-1 ; h=-N^{-1} \ln r, N>0-$ произвольное целое число. Очевидно, $\varrho_{j} \in(r, 1)$, причем $\varrho_{N}=r$, т.е. кольцо $K_{r}^{1}$ представляется как объединение колец $K_{\varrho_{j+1}}^{\varrho_{j}}, j=0,1, \ldots, N-1$.

Рассмотрим функции вида

$$
f^{N}(z)=\sum_{j=0}^{N-1} \varphi_{j}(\Theta) \chi_{j}(\varrho)
$$

где $(\varrho, \Theta)$ - полярные координаты, $\varphi_{j}(\Theta) \in L_{p}(-\pi, \pi)$ - произвольная функция, а $\chi_{j}(\varrho)$ характеристическая функция интервала $\left(\varrho_{j+1}, \varrho_{j}\right)$. Множество таких функций всюду плотно в $L_{p}\left(K_{r}^{1}\right)$.

Оценим вначале норму аналитической функции $f_{j \mathrm{a}}^{N}(z)=\left(\varphi_{j}(\Theta) \chi_{j}(\varrho)\right)_{\mathrm{a}}$. Имеем

$$
f_{j \mathrm{a}}^{N}(z)=\sum_{n=0}^{\infty} c_{n} z^{n}
$$

где, как показьвает подсчет,

$$
c_{n}=\frac{2(n+1)}{n+2} \varphi_{j n}\left(e^{-(n+2) j h}-e^{-(n+2)(j+1) h}\right),
$$


$\varphi_{j n}-$ коэффициенты Фурье функции $\varphi_{j}(\Theta)$. Таким образом,

$$
f_{j \mathrm{a}}^{h}(z)=\sum_{n=0}^{\infty} \widetilde{M}_{j n} \varphi_{j n} e^{i n \Theta}
$$

где

$$
\widetilde{M}_{j n}=\frac{2(n+1)}{n+2} \varrho^{n}\left(e^{-(n+2) j h}-e^{-(n+2)(j+1) h}\right) .
$$

Покажем, что последовательность

$$
M_{j n}=\varrho^{n}\left(e^{-(n+2) j h}-e^{-(n+2)(j+1) h}\right)
$$

удовлетворяет классической теореме Марцинкевича о мультипликаторах коэффициентов Фурье (см., например, [3, с. 346]).

ЗАмЕЧАнИЕ. Множители $2(n+1) /(n+2)$ не зависят от $j, h$ и $\varrho$ и поэтому роли не играют. Они, очевидно, образуют последовательность $L_{p}$-мультипликаторов коэффициентов Фурье, и мы можем применить теорему Марцинкевича повторно.

Нетрудно видеть, что производная функции

$$
M_{j x}=\frac{1}{\varrho^{2}}\left(e^{-(x+2)(j h-\ln \varrho)}-e^{-(x+2)(j h+h-\ln \varrho)}\right)
$$

обращается в нуль в точке $x$, удовлетворяющей уравнению

$$
e^{-(x+2) h}=\frac{j h-\ln \varrho}{j h+h-\ln \varrho} .
$$

Это уравнение имеет единственное решение $x>-2$ (локальный максимум). Следовательно, для всех $j=0,1, \ldots, n=0,1, \ldots, \varrho \in(r, 1)$

$$
\begin{aligned}
M_{j n} & \leqslant \frac{1}{\varrho^{2}}\left(\left(\frac{j h-\ln \varrho}{(j+1) h-\ln \varrho}\right)^{(j h-\ln \varrho) / h}-\left(\frac{j h-\ln \varrho}{(j+1) h-\ln \varrho}\right)^{((j+1) h-\ln \varrho) / h}\right) \\
& \leqslant \frac{1}{r^{2}}\left(\frac{j h-\ln \varrho}{(j+1) h-\ln \varrho}\right)^{(j h-\ln \varrho) / h}\left(1-\frac{j h-\ln \varrho}{(j+1) h-\ln \varrho}\right) \leqslant \frac{1}{r^{2}} \frac{h}{(j+1) h-\ln \varrho} .
\end{aligned}
$$

Так как функция $M_{j x}$ имеет только одну точку максимума, то при $2^{k} \leqslant n \leqslant 2^{k+1}-1$, $k=0,1, \ldots$, разности $M_{j, n+1}-M_{j n}$ меняют знак не более одного раза. Поэтому

$$
\sum_{n=2^{k}}^{2^{k+1}-1}\left|M_{j, n+1}-M_{j n}\right| \leqslant \frac{4 h}{r^{2}((j+1) h-\ln \varrho)}
$$

Таким образом, по теореме Марцинкевича

$$
\left\|f_{j \mathrm{a}}^{N}(z)\right\|_{L_{p}(-\pi, \pi)} \leqslant M \frac{h}{(j+1) h-\ln \varrho}\left\|\varphi_{j}(\Theta)\right\|_{L_{p}(-\pi, \pi)},
$$

где $M>0$ - постоянная, зависящая от $r$. 
Отсюда находим, что для функции $f_{\mathrm{a}}^{N}(z)$ имеем

$$
\left\|f_{\mathrm{a}}^{N}(z)\right\|_{L_{p}(-\pi, \pi)} \leqslant M \sum_{j=0}^{N-1} \frac{h}{(j+1) h-\ln \varrho}\left\|\varphi_{j}(\Theta)\right\|_{L_{p}(-\pi, \pi)} .
$$

Далее, заметив, что в соответствии с выбором чисел $\varrho_{j}, j=0,1, \ldots, N-1$, имеем неравенство $\varrho_{j}^{2}-\varrho_{j+1}^{2} \geqslant 2 r^{2} h$, и, следовательно, из оценки (21) получаем, что

$$
\left\|f_{\mathrm{a}}^{N}(z)\right\|_{L_{p}(-\pi, \pi)} \leqslant M \sum_{j=0}^{N-1} \frac{h^{1 / p^{\prime}}}{(j+1) h-\ln \varrho}\left\|\varphi_{j}(\Theta)\right\|_{L_{p}(-\pi, \pi)}\left(\varrho_{j}^{2}-\varrho_{j+1}^{2}\right)^{1 / p} .
$$

Отсюда, используя неравенство Гёльдера для сумм, получаем неравенство

$$
\begin{aligned}
\left\|f_{\mathrm{a}}^{N}(z)\right\|_{L_{p}(-\pi, \pi)} \leqslant & M h^{1 / p^{\prime}}\left(\sum_{j=0}^{N-1} \frac{1}{((j+1) h-\ln \varrho)^{p^{\prime}}}\right)^{1 / p^{\prime}} \\
& \times\left(\sum_{j=0}^{N-1}\left\|\varphi_{j}(\Theta)\right\|_{L_{p}(-\pi, \pi)}^{p} \frac{\varrho_{j}^{2}-\varrho_{j+1}^{2}}{2}\right)^{1 / p} \\
\leqslant & M h^{1 / p^{\prime}} \frac{1}{h-\ln \varrho}\left\|f^{N}(z)\right\|_{L_{p}\left(K_{r}^{1}\right)}
\end{aligned}
$$

или, что то же,

$$
\left\|f_{\mathrm{a}}^{N}(z)\right\|_{L_{p}(-\pi, \pi)}^{p} \leqslant M h^{p-1} \frac{1}{(h-\ln \varrho)^{p}}\left\|f^{N}(z)\right\|_{L_{p}\left(K_{r}^{1}\right)}^{p} .
$$

Интегрируя последнее неравенство по $\varrho$ от $r$ до 1 , получаем искомое неравенство

$$
\left\|f_{\mathrm{a}}^{N}(z)\right\|_{L_{p}\left(K_{r}^{1}\right)}^{p} \leqslant M\left\|f^{N}(z)\right\|_{L_{p}\left(K_{r}^{1}\right)}^{p} .
$$

Предложение 4 доказано.

\section{СПИСОК ЦИТИРОВАННОЙ ЛИТЕРАТУРЫ}

[1] Axler Sh. Bergman spaces and their applications // Surveys of Some Recent Results in Operator Theory / ed. J. Conway, B. Morell. Pitman Res. Notes Math. Ser. V. 1. Harlow: Longman Sci. Tech., 1988. P. 1-50.

[2] Бари Н. К. Тригонометрические ряды. М.: Наука, 1961.

[3] Шведенко С. В. Классы Харди и связанные с ними пространства аналитических функций // Итоги науки и техн. Матем. анализ. Т. 23. М.: ВИНИТИ, 1985. С. 3-124.

[4] Зигмунд А. Тригонометрические ряды. Т. 2. М.: Физматгиз, 1965. 